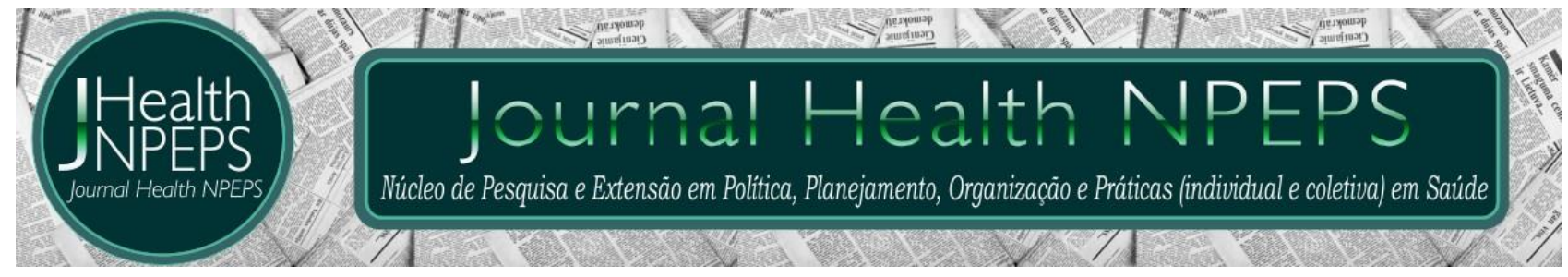

http://dx.doi.org/10.30681/252610104626

ENSAIO TEÓRICO-REFLEXIVO

\title{
Planejamento organizacional no contexto de pandemia por COVID-19: implicações para a gestão em enfermagem
}

\section{Organizational planning in pandemic context by COVID-19: implications for nursing management}

\section{Planificación organizativa en el contexto pandémico por COVID-19: implicaciones para la gestión de enfermería}

\author{
João Miguel Almeida Ventura-Silva1, Olga Maria Pimenta Lopes Ribeiro², Margarida \\ Reis Santos 3 , Ana da Conceição Alves Faria ${ }^{4}$, Maria Amélia José Monteiro ${ }^{5}$, \\ Lara Vandresen ${ }^{6}$
}

\begin{abstract}
RESUMO
Objetivo: refletir sobre o planejamento organizacional no contexto da pandemia por COVID-19 e as implicações para a gestão em enfermagem. Método: estudo téoricoreflexivo, realizado entre os dias 01 e 09 de junho de 2020 e baseado nos documentos emanados pela Direção Geral da Saúde de Portugal para o planejamento organizacional no contexto hospitalar, durante o período de 03 de março a 03 de junho de 2020. Resultados: foram identificados nove documentos do tipo normas ou orientações emitidas pela Direção Geral da Saúde, referentes às medidas para controle da pandemia
\end{abstract}

\footnotetext{
${ }^{1}$ Licenciado em Enfermagem. Doutorando em Ciências de Enfermagem. Enfermeiro Especialista em Enfermagem de Reabilitação no Centro Hospitalar Universitário São João. Porto, Portugal. E-mail: enf.joao.ventura@gmail.com ORCID ID: http://orcid.org/0000-0002-8794-528X Endereço para correspondência: Rua Companhia dos Caulinos, 358, 3으 Direito Traseiras, 4460-205 Senhora da Hora. Porto, Portugal.

${ }^{2}$ Licenciada em Enfermagem. Mestre e Doutora em Ciências de Enfermagem. Professora Adjunta na Escola Superior de Enfermagem do Porto. Investigadora doutorada integrada no Centro de Investigação em Tecnologias e Serviços de Saúde (CINTESIS). Porto, Portugal. E-mail: olgaribeiro@esenf.pt ORCID ID: http://orcid.org/0000-0001-9982-9537

${ }^{3}$ Licenciada em Enfermagem. Mestre e Doutora em Ciências de Enfermagem. Professora Coordenadora na Escola Superior de Enfermagem do Porto. Investigadora integrada no Centro de Investigação em Tecnologias e Serviços de Saúde (CINTESIS). Porto, Portugal. E-mail: mrs@esenf.pt ORCID ID: http://orcid.org/0000-0002-7948-9317

${ }^{4}$ Licenciada em Enfermagem. Doutoranda em Ciências de Enfermagem. Enfermeira Especialista em Enfermagem de Reabilitação na Unidade de Cuidados da Comunidade Terras de Camilo do Agrupamento de Centros de Saúde Ave/Famalicão. Vila Nova de Famalicão, Portugal. E-mail: anafaria85@msn.com ORCID ID: http://orcid.org/0000$\underline{0002-5838-0080}$

${ }^{5}$ Licenciada em Enfermagem. Mestre em Ciências de Enfermagem. Docente na Universidade Fernando Pessoa, Departamento de Enfermagem. Porto, Portugal. E-mail: ajose@ufp.edu.pt ORCID ID: http://orcid.org/0000-00024774-0554

${ }^{6}$ Enfermeira. Doutoranda em Enfermagem pela Universidade Federal de Santa Catarina (UFSC). Florianópolis, Santa Catarina, Brasil. E-mail: laravandresen@hotmail.com ORCID ID: http://orcid.org/0000-0002-1389-7932
} 
da COVID-19, sendo possível a organização dos dados em duas macrocategorias: estruturas, materiais e procedimentos e exercício profissional dos enfermeiros, com enfoque no papel do enfermeiro gestor. Conclusão: a Direção-Geral da Saúde de Portugal, através da disseminação de documentos orientadores, permitiu um planejamento organizacional, ajustado às necessidades dos pacientes com COVID-19. 0 enfermeiro gestor teve de reorganizar a gestão de estruturas e materiais, assim como a gestão dos enfermeiros para cumprir as orientações emanadas e continuar a assegurar cuidados de qualidade aos pacientes internados em sua unidade.

Descritores: Infecções por Coronavírus; Gestão em Saúde; Diretrizes para o Planejamento em Saúde; Enfermagem.

\section{ABSTRACT}

Objective: to reflect on organizational planning in the context of the COVID-19 pandemic and the implications for nursing management. Method: theoretical-reflective study, carried out between 01 and 09 June 2020 and based on documents issued by the General Directorate of Health of Portugal for organizational planning in the hospital context, during the period from March 03 to June 03, 2020. Results: nine documents of the norms or guidelines issued by the Directorate-General for Health were identified, referring to the measures to control the pandemic of COVID-19, making it possible to organize the data in two macro categories: structures, materials and procedures and professional practice of nurses, focusing on the role of nurse manager. Conclusion: the Directorate-General for Health of Portugal, through the dissemination of guiding documents, allowed for organizational planning, adjusted to the needs of patients with COVID-19. The nurse manager had to reorganize the management of structures and materials, as well as the management of nurses to comply with the guidelines issued and continue to ensure quality care for patients admitted to his unit.

Descriptors: Coronavirus Infections; Health Management; Health Planning Guidelines; Nursing.

\section{RESUMEN}

Objetivo: reflexionar sobre la planificación organizacional en el contexto de la pandemia COVID-19 y las implicaciones para el manejo de enfermería. Método: estudio teórico-reflexivo, realizado entre el 01 y el 09 de junio de 2020 y basado en documentos emitidos por la Dirección General de Salud de Portugal para la planificación organizativa en el contexto hospitalario, durante el período del 03 de marzo al 03 de junio. 2020. Resultados: se identificaron nueve documentos de las normas o directrices emitidas por la Dirección General de Salud, en relación con las medidas para controlar la pandemia de COVID-19, que permiten organizar los datos en dos macro categorías: estructuras, materiales y procedimientos y práctica profesional. de enfermeras, centrándose en el papel de gerente de enfermería. Conclusión: la Dirección General de Salud de Portugal, a través de la difusión de documentos guía, permitió la planificación organizacional, ajustada a las necesidades de los pacientes con COVID-19. El gerente de enfermería tuvo que reorganizar la gestión de estructuras y materiales, así como la gestión de las enfermeras para cumplir con las directrices emitidas y continuar garantizando una atención de calidad para los pacientes ingresados en su unidad.

Descriptores: Infecciones por Coronavirus; Gestión en Salud; Directrices para la Planificación en Salud; Enfermería.

\section{INTRODUÇÃO}


O início da década de 2020 ficou marcado pela pandemia do novo coronavírus, que provoca a severe acute respiratory syndrome, designado por SARS-CoV-2, afetando pessoas de todas as nações, continentes, raças e grupos socioeconômicos. Sendo, por isso, uma das crises centrais de saúde de uma geração ${ }^{1}$. Tal fato, conduziu o diretor da Organização Mundial de Saúde, em 11 de março de 2020, a declarar o estado de emergência pública².

Desde o início do ano até 03 de junho de 2020, já foram confirmados em todo o mundo 6.287.547 casos da COVID19, com maior prevalência nos Estados Unidos da América (3.311.387 infectados), seguidos pela Europa, com 2.268.311 pessoas infectadas ${ }^{3}$.

Em Portugal, até dia 03 de junho de 2020, data em que se completaram três meses desde o primeiro caso de COVID-19, registou-se o total de 32.895 casos de infecção ${ }^{4}$. Ao longo deste período de tempo, o número de internações por COVID-19 atingiu o pico máximo diário em 16 de abril com 1302 episódios. A maior taxa de internações em Unidades de Cuidados Intensivos (UCI), foi registada no dia 07 de abril, com 271 internamentos. Verificou-se uma prevalência significativa, no que concerne às internações hospitalares pela COVID-19 durante o mês de maio, havendo, posteriormente, uma diminuição considerável até ao dia 03 de junho de 2020, estando nessa data internados em instituições hospitalares portuguesas 445 pacientes, dos quais 58 estavam em $\mathrm{UCl}^{5}$.

Durante todo este tempo, tal como em muitos outros países, foi fundamental a sintonia na ação entre políticos, gestores e chefias de saúde. Assim, para além de todas as decisões/orientações governamentais, importa salientar as estratégias locais e ajustadas às populações ${ }^{6,7}$, que permitiram uma resposta adequada de cuidados. Verdadeiramente, às instituições de saúde foi exigido um planejamento organizacional em tempo diminuto, no sentido de atender a necessidade de recursos materiais (aquisição de insumos e de equipamento) e humanos (organização dos profissionais e das equipes), criação de intervenções integradas e definição de vários planos de ação em situação de contingência ${ }^{8}$.

Em todo este processo de reestruturação hospitalar, a gestão em enfermagem foi fundamental. Nesta nova fase, para o Sistema Nacional de Saúde, que motivou a tomada de medidas urgentes e que mudaram de forma significativa o cotidiano dos 
profissionais de saúde $^{9}$, o enfermeiro gestor teve um papel relevante ao incorporar na gestão da sua unidade, as novas orientações do órgão regulador da saúde, de modo a responder às solicitações, no âmbito do combate à COVID-19. Nos vários contextos, a promoção do trabalho em equipe, pelo enfermeiro gestor, permitiu valorizar os cuidados de enfermagem centrados na pessoa ${ }^{10}$.

Atendendo ao apresentado e à relevância para a enfermagem, enquanto profissão e disciplina, este estudo tem como objetivo refletir sobre 0 planejamento organizacional no contexto da pandemia por COVID-19 e as implicações para a gestão em enfermagem.

\section{MÉTODO}

Estudo teórico-reflexivo, baseado na documentação produzida pela DGS de Portugal. A coleta de dados ocorreu no período compreendido entre os dias 01 e 09 de junho de 2020, no site oficial da DGS. Os critérios de inclusão na amostra foram os documentos do tipo normas ou as orientações da DGS, referentes à pandemia da COVID-19, publicadas no período de 03 de março a 03 de junho 2020.

No tratamento dos dados, realizou-se análise temática, segundo o referencial de Bardin ${ }^{11}$. Efetuou-se a pré-análise, exploração e tratamento dos achados. Dois revisores independentes realizaram a avaliação crítica, extração e síntese dos dados. Qualquer discordância foi resolvida, através de discussão ou recurso a um terceiro revisor. Foram identificados catorze documentos. Com base na leitura de título e assunto, foram excluídos cinco documentos, por estarem relacionados com a Atenção Primária à Saúde, passando nove documentos para análise integral.

Os dados extraídos dos documentos selecionados foram compilados, em um quadro desenvolvido especificamente para o estudo, e agrupados por: título/data de publicação, assunto, recomendações para a prática clínica e contributo para os temas. 
Quadro 1 - Dados extraídos da análise dos documentos selecionados.

\begin{tabular}{|c|c|c|c|}
\hline $\begin{array}{l}\text { Título/ } \\
\text { Data }\end{array}$ & Assunto & Recomendações para a prática clínica & $\begin{array}{l}\text { Contributos } \\
\text { para o tema }\end{array}$ \\
\hline $\begin{array}{l}\text { Norma } \\
001 / 2020\end{array}$ & $\begin{array}{l}\text { COVID-19: Primeira fase de } \\
\text { mitigação } \quad-\quad \text { medidas } \\
\text { transversais de preparação. }\end{array}$ & $\begin{array}{l}\text { Informar sobre as medidas de mitigação que } \\
\text { garantam a adequação e sustentabilidade } \\
\text { do sistema de saúde. }\end{array}$ & $\begin{array}{l}\text { Estruturas, } \\
\text { materiais e } \\
\text { procedimentos. }\end{array}$ \\
\hline $\begin{array}{l}\text { Norma } \\
004 / 2020\end{array}$ & $\begin{array}{l}\text { COVID-19: Fase de } \\
\text { mitigação - abordagem em } \\
\text { suspeita/infecção }\end{array}$ & $\begin{array}{l}\text { Evidenciar o modelo de abordagem do } \\
\text { paciente com suspeita ou infecção por SARS- } \\
\text { CoV-2, aplicável às unidades de saúde. }\end{array}$ & $\begin{array}{l}\text { Estruturas, } \\
\text { materiais } \\
\text { procedimentos. }\end{array}$ \\
\hline $\begin{array}{l}\text { Norma } \\
005 / 2020\end{array}$ & 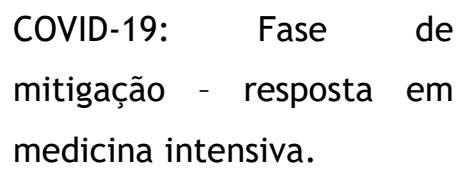 & $\begin{array}{l}\text { Fornecer orientações sobre a assistência de } \\
\text { pacientes internados em medicina intensiva. }\end{array}$ & $\begin{array}{l}\text { Estruturas, } \\
\text { materiais } \quad \mathrm{e} \\
\text { procedimentos. }\end{array}$ \\
\hline $\begin{array}{l}\text { Norma } \\
007 / 2020\end{array}$ & $\begin{array}{l}\text { Prevenção e controle de } \\
\text { infecção por SARS-CoV-2 } \\
\text { (COVID-19): EPI. }\end{array}$ & $\begin{array}{l}\text { Definir a adequada utilização de EPI, pelos } \\
\text { profissionais de saúde. }\end{array}$ & $\begin{array}{l}\text { Estruturas, } \\
\text { materiais e } \\
\text { procedimentos. }\end{array}$ \\
\hline $\begin{array}{l}\text { Norma } \\
012 / 2020\end{array}$ & $\begin{array}{l}\text { COVID-19: exames } \\
\text { endoscópicos digestivos. }\end{array}$ & $\begin{array}{l}\text { Fornecer orientações sobre priorização dos } \\
\text { procedimentos endoscópicos e as medidas } \\
\text { de prevenção e controle de infecção na } \\
\text { realização das técnicas de gastrenterologia. }\end{array}$ & $\begin{array}{l}\text { Estruturas, } \\
\text { materiais e } \\
\text { procedimentos. }\end{array}$ \\
\hline $\begin{array}{l}\text { Orientação } \\
013 / 2020\end{array}$ & $\begin{array}{l}\text { Profissionais de saúde com } \\
\text { exposição a SARS-CoV-2 } \\
\text { (COVID-19). }\end{array}$ & $\begin{array}{l}\text { Evidenciar orientações sobre a identificação } \\
\text { precoce de sintomas nos profissionais de } \\
\text { saúde e definição das medidas de controle } \\
\text { da infecção e de prevenção adequadas. }\end{array}$ & $\begin{array}{l}\text { Exercício } \\
\text { profissional dos } \\
\text { enfermeiros. }\end{array}$ \\
\hline $\begin{array}{l}\text { Orientação } \\
018 / 2020\end{array}$ & COVID-19: gravidez e parto. & $\begin{array}{l}\text { Realçar a necessidade de redimensionar } \\
\text { recursos, aumentar a segurança assistencial } \\
\text { a mulheres infetadas e conter cadeias de } \\
\text { transmissão. }\end{array}$ & $\begin{array}{l}\text { Exercício } \\
\text { profissional dos } \\
\text { enfermeiros. }\end{array}$ \\
\hline $\begin{array}{l}\text { Norma } \\
008 / 2020\end{array}$ & $\begin{array}{l}\text { COVID-19: Fase de } \\
\text { mitigação - pacientes com } \\
\text { doença renal crônica em } \\
\text { hemodiálise. }\end{array}$ & $\begin{array}{l}\text { Orientar a abordagem clínica dos pacientes } \\
\text { com suspeita e infecção confirmada por } \\
\text { SARS-CoV-2 no sistema de saúde, com } \\
\text { doença renal crônica em diálise. }\end{array}$ & $\begin{array}{l}\text { Exercício } \\
\text { profissional dos } \\
\text { enfermeiros. }\end{array}$ \\
\hline $\begin{array}{l}\text { Norma } \\
009 / 2020\end{array}$ & $\begin{array}{l}\text { COVID-19: Fase de } \\
\text { mitigação reconfiguração } \\
\text { dos cuidados de saúde na } \\
\text { área da oncologia. }\end{array}$ & $\begin{array}{l}\text { Facilitar a abordagem clínica dos pacientes, } \\
\text { da área da oncologia, com suspeita e } \\
\text { infecção confirmada por SARS-CoV-2 no } \\
\text { sistema de saúde. }\end{array}$ & $\begin{array}{l}\text { Exercício } \\
\text { profissional dos } \\
\text { enfermeiros. }\end{array}$ \\
\hline
\end{tabular}

\section{RESULTADOS E DISCUSSÃO}


A amostra final do estudo foi constituída por nove documentos. Após realização de análise temática, identificaram-se dois temas: estruturas, materiais e procedimentos e exercício profissional dos enfermeiros.

Considerando a rápida transmissibilidade do SARS-CoV-2 no seio da população, a criação de procedimentos, no sentido de manter a integridade da rede de saúde pública, através do planejamento, treinamento dos profissionais envolvidos, e a eliminação de potenciais ou efetivos riscos de contágio do vírus, tornou-se uma preocupação constante do órgão governamental e alerta frente aos impactos.

\section{Estruturas, materiais e procedimentos}

Face à necessidade premente da reorganização dos materiais e estruturas afetados à prestação de cuidados de saúde dos pacientes com COVID-19, foi necessário implementar várias alterações nas instituições de saúde.

No que concerne aos hospitais, em cada serviço de urgência, foi necessária a criação de áreas dedicadas a avaliação e tratamento destes pacientes, apresentando-se com sinalética adequada, e a definição de circuitos internos e áreas de internamento, em isolamento, perante casos confirmados ou suspeitos, garantindo a separação de pacientes. 0 equipamento e material de consumo clínico foi organizado por médicocirúrgico, de proteção individual, material de consumo clínico e outros equipamentos $^{12-14}$. A propósito do apresentado, em um relato de experiência de um hospital universitário de Portugal, no sentido de uma resposta integrada da instituição, houve preocupação pela aquisição de equipamentos e dotação de material clínico para os vários serviços envolvidos nesta pandemia e o estabelecimento de vários planos de ação, no contexto da COVID-198. O mesmo também foi realizado em um hospital do interior de Portugal, que esteve dedicado ao cuidado destes pacientes ${ }^{7}$.

Esta pandemia, além da reformulação nos contextos de urgência e internamento, exigiu uma resposta dos serviços de medicina intensiva, através da reorganização das suas atividades e da disponibilização das equipes clínicas, para atividade assistencial nas $\mathrm{UCl}$. Assim, procedeu-se a ativação de todos os leitos anteriormente inativados, para pacientes críticos e em espaços contíguos aos atuais serviços de 
medicina intensiva, evitando a dispersão dos profissionais de saúde; a conversão do número adequado de leitos destinados a pacientes críticos no contexto de cada unidade hospitalar; e por fim, a criação de áreas dedicadas a prestação de cuidados em fim de vida ${ }^{15}$. No contexto das UCI dedicadas a COVID19, tornou-se fundamental a separação de pacientes infectados e pacientes com elevado índice de suspeita clínica, em locais específicos, muitos deles com possibilidade de pressão negativa ${ }^{8}$. Esta realidade não foi apenas vivenciada em Portugal, sendo também relatada na Espanha, em um plano de contingência para serviços de terapia intensiva direcionados à pandemia da COVID-1916. Também no Brasil, através de uma nota técnica, é exposta a necessidade de se otimizar o uso dos serviços existentes e dimensionar recursos que serão fundamentais para fortalecer a capacidade de resposta do sistema de saúde ${ }^{17}$.

Reforça neste contexto a estratégia global de gestão, acesso e utilização de Equipamentos de Proteção Individual (EPI). No âmbito da COVID-19, deverá ser garantido o fornecimento adequado e suficiente de EPI aos profissionais e constituir uma reserva estratégica local que corresponda às necessidades que emergem em contexto de pandemia. $O$ uso de EPI está relacionado com o risco de exposição e a dinâmica de transmissão do vírus. Assim sendo, no decorrer da pandemia o uso de máscara pelos profissionais, passou a ser obrigatório no interior das instituições de saúde e no momento de observação ou contato com pacientes suspeitos ou confirmados com COVID-19, acrescentando as restantes precauções adicionais consoante a indicações específicas $^{18}$.

Deste modo, salienta-se o papel fundamental da gestão, nomeadamente, da gestão em enfermagem, no que concerne ao seu contributo na criação de estratégias de racionalização dos materiais e a promoção da capacitação dos profissionais da equipe, no sentido da correta colocação, utilização e remoção de EPI e deteção precoce de eventual caso de COVID-197,10,19. Esta mesma situação é corroborada no contexto internacional, reforçando a necessidade de coordenar a cadeia de fornecimento dos EPI, implementar estratégias que minimizem a necessidade desadequada de EPI e garantir o seu uso de forma ajustada ${ }^{16,20}$.

Ainda nesta área temática foi possível identificar uma norma relacionada com a realização de 
procedimentos, que também aborda conteúdos sobre as estruturas e materiais. 0 documento fornece orientações quanto à realização de procedimentos endoscópicos no tubo digestivo alto ${ }^{21}$. Atendendo a esta norma, houve a necessidade da priorização clínica dos exames a realizar, com a salvaguarda da monitorização regular da evolução da pandemia no contexto português, disponibilidade de recursos humanos e materiais, sem prejuízo da avaliação clínica de cada caso. Salienta também alguns aspectos relacionados com a circulação condicionada de profissionais nos serviços onde se realizam estas técnicas, a criação de uma sala destinada a pacientes com COVID-19, equipada com pressão negativa sendo todos os equipamentos e materiais exclusivos a esse espaço. Por outro lado, é feita alusão à utilização de EPI e medidas que visem a prevenção e controle da infecção, designadamente o uso de fato dentro das unidades de gastroentereologia, a adoção de distanciamento físico adequado, bem como a higienização das mãos sempre que aplicável ${ }^{21}$.

Num estudo teórico desenvolvido em Portugal ${ }^{22}$, acerca da realização de procedimentos endoscópicos em tempos de pandemia por COVID-19, os autores reforçam a importância do adiamento de procedimentos considerados eletivos, pois essa medida reduz a concentração de pessoas nos hospitais e a carga de trabalho dos profissionais, permitindo um menor risco de transmissão no momento da realização do procedimento. Salientam ainda a importância da criação de salas com pressão negativa para a realização do exame e o uso racionado de recursos materiais, pois os profissionais de saúde presentes nas salas de endoscopia correm um risco maior de infecção devido à inalação de gotículas no ar, contato conjuntival e a contaminação por toque.

A nível internacional, concretamente na realidade brasileira, vários autores advertem para a reavaliação da necessidade de execução dos procedimentos endoscópicos, referindo que apenas devem ser realizados os urgentes. No que concerne aos profissionais de saúde, a adoção rigorosa de medidas de proteção é fundamental ${ }^{23}$.

No contexto da gestão em enfermagem, o enfermeiro gestor desempenha um papel fundamental na prevenção da transmissão do SARS-CoV2 , quer entre pacientes e mesmo entre 
profissionais. Por outro lado, mais do que nunca, na atual pandemia, o enfermeiro gestor, ao assegurar a gestão dos recursos humanos disponíveis, otimizando a sua eficiência, eficácia e produtividade, torna-se imprescindível para uma melhor assistência aos pacientes e uma maior satisfação dos profissionais $^{19}$.

\section{Exercício profissional dos enfermeiros}

Todos os gestores, de acordo com seu conhecimento e competências, contribuíram para a elaboração de planos de contingência dos hospitais, assim como adequação das normas emanadas pela DGS ao contexto de cada serviço e especificidades clínicas, conforme as necessidades e política hospitalar. Em relação ao enfermeiro gestor, este teve como funções primordiais assegurar a gestão e organização dos cuidados, promovendo a segurança do paciente e dos profissionais que trabalham nos serviços.

Deste modo, para que sejam prestados cuidados seguros, gerindo 0 risco de infecção por COVID-19 e prevenindo infecções cruzadas, o enfermeiro gestor teve que promover práticas baseadas em evidência, dar formação e integrar novos enfermeiros, assegurar dotações seguras, atendendo à complexidade dos cuidados. Segundo o seu perfil de competências, cabe ao enfermeiro gestor organizar os serviços, promovendo cuidados de saúde de excelência, sem esquecer os riscos que esse cuidado de primeira linha acarreta para os enfermeiros e restantes profissionais de saúde.

0 enfermeiro gestor não só promove o cumprimento e interpretação correta das normas, como gere os riscos dos profissionais associados aos cuidados de saúde, em relação aos aspectos psicológicos e desgastes, não só pelo medo do próprio adoecimento ${ }^{24}$, como pela vivência das angústias e óbitos dos pacientes e pares.

Os enfermeiros são profissionais presentes em momentos mais preciosos e alguns mais trágicos, de acordo com a essência da profissão, servem a humanidade, protegendo a saúde e o bem-estar dos indivíduos, comunidades e nações ${ }^{25}$, aspecto também revelado no presente contexto pandêmico. 0 enfermeiro gestor detém um conhecimento concreto e uma visão da organização que the permite identificar os fatores contingenciais que interferem nas atividades de planejamento, execução, controle e avaliação. É o responsável, em primeira linha, pela 
defesa da segurança e qualidade dos cuidados de enfermagem assumindo um papel de relevo no contexto da pandemia ${ }^{19}$.

É sabido que os enfermeiros são os profissionais de saúde que apresentam maior risco de exposição ao SARS-CoV-2, pois são aqueles que estão na linha de frente da assistência, liderando políticas de humanização e desenvolvendo cuidados com níveis de complexidade distintos e indissociáveis, desde uma simples ação educativa sobre lavagem das mãos até práticas de cuidado complexo ${ }^{26,27}$.

Desta forma, o enfermeiro gestor desempenha um papel crucial na adoção de todas as recomendações de prevenção e controle de infecção, incluindo o uso de EPI, assim como a monitorização da temperatura corporal e a identificação precoce de sinais e sintomas nestes profissionais é imprescindível, no sentido de garantir a segurança de todos as pessoas que se encontram nas instituições de saúde. Assim, para além da adoção do uso de máscara cirúrgica por todos os profissionais, foi também definido o conceito de caso suspeito, quando o profissional apresenta sintomatologia compatível com a COVID-19, bem como classificado o tipo de contato próximo (alto risco ou baixo risco de exposição) e a sua abordagem. A necessidade de identificação de contatos próximos, a preocupação para limitar o número de profissionais de saúde expostos a pacientes com COVID-19 e privilegiar equipes exclusivas dedicadas à prestação de cuidados são também mencionadas ${ }^{28}$.

Estudo lusitano evidenciou o cumprimento do uso de máscara por todos os profissionais e pacientes internados; a tentativa de reduzir o número de pessoas a circularem no hospital, sendo apenas autorizadas as visitas aos pacientes em situações especiais, como é o caso de pacientes em fim de vida; e a experiência positiva da criação de um modelo de equipes mistas, constituídas por elementos de várias especialidades médicas, destacadas em cada dia, para cada uma das áreas dedicadas à prestação de cuidados aos pacientes com COVID-198.

Os três documentos restantes divulgados pela DGS estavam relacionados com contextos específicos da prestação de cuidados. Em 30 de março é apresentada uma orientação dirigida à gravidez e parto, no contexto da COVID-19, que apresenta os critérios de caso e de cura; os cuidados prénatais, particularmente a organização dos serviços e proteção dos profissionais; 
os cuidados de vigilância da gravidez na mulher com e sem COVID-19; os critérios para o internamento da mulher, com e sem COVID-19, durante a gravidez e os cuidados obstétricos; e, finalmente, as orientações relacionados com 0 internamento para a assistência ao parto. São também abordados os aspectos relacionados com 0 acompanhamento da grávida durante o parto, realçando que é um direito reconhecido nos serviços de saúde, mas face ao atual contexto de pandemia, foram definidos um conjunto de critérios a ser obedecidos, no sentido de usufruir desse direito ${ }^{29}$.

Uma revisão sistemática da literatura, realizada no Brasil, apresentou aspectos concordantes com as orientações adotadas em Portugal, em específico sobre a importância dos cuidados pré-natais e obstétricos, recomendações quanto à admissão da mulher na instituição de saúde, ao trabalho de parto e puerpério. Acrescenta ainda aspectos relacionados com os cuidados aos recém-nascidos, filhos de mulheres com diagnóstico de COVID-1930.

A DGS emanou ainda uma norma referente aos pacientes com doença renal crônica em diálise. É dada ênfase às medidas de prevenção e controle nas
Unidades de Hemodiálise; a informação aos pacientes sobre sintomas sugestivos de COVID-19; a organização e gestão das unidades de hemodiálise, quer em recursos materiais, quer em recursos humanos; e a abordagem clínica dos pacientes em hemodiálise com suspeita ou confirmação de COVID-1931. No âmbito da prestação de cuidados aos pacientes com doença renal crônica, alguns autores sustentam que se vivem tempos difíceis com a atual pandemia, o que obrigou a uma total reformulação dos cuidados na área da saúde, em particular na especialidade de nefrologia. No contexto da hemodiálise, os cuidados são prestados em grupo, com afluência frequente e não dispensável, incrementando um inevitável contato com profissionais de saúde e outros usuários, pelo que se torna necessária a adoção de todas as medidas que visem o combate à infecção 32 .

Foi identificado um documento, direcionado à reconfiguração dos cuidados de saúde na área da oncologia, dando ênfase às medidas gerais de prevenção e controle da infecção, com a reorganização das equipes de profissionais de saúde e previsão da redução da força de trabalho, a possibilidade de realizar atos clínicos com recurso à teleconsulta e 
telemonitorização; à organização da prestação de cuidados, com enfoque na criação de circuitos separados fisicamente da restante atividade assistencial; ao rastreio dos pacientes para testar a COVID-19; e a gestão de pacientes oncológicos com COVID-1933, sendo estas orientações corroboradas pela literatura internacional. Um estudo brasileiro salienta a necessidade da organização dos espaços e fluxos de atendimento, assim como oferecer aos pacientes a modalidade de teleatendimento, como estratégia de acompanhamento, permitindo a comunicação entre os pacientes e a equipe de saúde e evitando as ídas não necessárias às instituições. Por outro lado, aponta como fundamental o reconhecimento precoce dos indivíduos infectados, com vista ao sucesso no tratamento e na redução da transmissibilidade ${ }^{27}$.

Face ao exposto, verificou-se uma reconfiguração de todas as estruturas das instituições de saúde, a responder às necessidades de cuidados dos pacientes com COVID-19. $\mathrm{Na}$ verdade, esta reestruturação só foi eficaz, devido a capacidade de adaptação dos profissionais às funções que vão sendo criadas e alteradas, considerando as necessidades, que surgem em cada momento ${ }^{8}$. Neste sentido, a existência de um modelo assistencial estruturado por equipes multidisciplinares e a liderança dessas equipes, permitiu uma capacidade de resposta eficaz às necessidades surgidas com a pandemia. Foi notória a partilha de conhecimento e a criação de um espírito de grupo entre os vários profissionais, imprescindíveis ao sucesso nesse momento 7,8 .

No que concerne aos enfermeiros, os fatores apresentados anteriormente foram cruciais para o desempenho profissional. A necessidade de reestruturação dos serviços clínicos nas instituições hospitalares, com a suspensão de toda a atividade cirúrgica programada mantendo apenas a urgente ${ }^{34}$, resultou na disponibilidade de profissionais de enfermagem para reforçar as equipes dos serviços, com todo seu arsenal terapêutico, assistencial ${ }^{35}$ e gerencial. Desta forma, o fato de estes profissionais terem que integrar essas equipes, com as quais nunca trabalharam, tendo apenas em comum a experiência de outrora em unidades de cuidados intensivos e serviços de internamento médicos, mobilizou esforços individuais às adaptações, que só foram possíveis pela 
determinação, alto profissionalismo e compromisso ético.

Neste contexto, o enfermeiro gestor, enquanto líder nas várias unidades e serviços que gere, desempenha um papel de destaque na gestão dos recursos disponíveis e na atenção às equipes que cuidam de pacientes com COVID-19 que exigem cuidados complexos ${ }^{10}$. Efetivamente, cabe ao enfermeiro gestor desempenhar com responsabilidade a liderança técnica capaz de considerar as diversas necessidades resultantes da crise, com o envolvimento dos vários segmentos da instituição ${ }^{27}$.

Por outro lado, considerando que o conhecimento sobre os aspectos relacionados com o comportamento deste vírus se encontra em constante mudança, a necessidade de acesso a fontes confiáveis e a capacitação dos enfermeiros, nos vários contextos da prática é inquestionavelmente importante, através da criação de momentos formativos e de reflexão sobre as práticas, de forma a promover 0 empenho da equipe na gestão das suas próprias competências ${ }^{18,32,36}$, aspecto fundamental nesta crise pandémica.

\section{CONCLUSÃO}

A pandemia por COVID-19 veio mudar o rumo da humanidade e marcar a história. Com o surgimento da pandemia, foram adotadas, a nível social, um conjunto de atividades planejadas e organizadas com o objetivo de diminuir o número de casos de contágio do vírus, protegendo os grupos mais vulneráveis. A par dessa resposta social, tornou-se imprescindível a resposta coordenada e eficaz das várias áreas governamentais, no que concerne à dotação de equipamentos, materiais e recursos humanos, bem como à elaboração de documentos orientadores para a prestação dos cuidados de saúde.

In loco, o planejamento organizacional no combate à COVID-19 foi fundamental, baseado nas orientações da DGS, permitindo a tomada de decisões baseadas na melhor evidência científica disponível. No que concerne aos enfermeiros, o papel de gestor foi relevante, na garantia pelo cumprimento das melhores práticas de reorganização de estruturas, de controle de infecção e de segurança, mas um realce importante na gestão das pessoas, no acompanhamento das medidas definidas pelos órgãos de gestão e na comunicação com as equipes e com os pacientes. O seu papel foi igualmente importante na informação transmitida à 
equipe, através do debate e transmissão de informações sobre a evolução da situação da COVID-19 na unidade/hospital e sobre as normas e medidas que devem ser adotadas para prevenir a contaminação.

\section{REFERÊNCIAS}

1. Shanafelt T, Ripp J, Trockel M. Understanding and addressing sources of anxiety among health care professionals during the COVID-19 pandemic. Jama. 2020; 323(21):21332134.

2. Organização Pan-Americana da Saúde. OMS afirma que COVID-19 é agora caracterizada como pandemia. Brasília; 2020 [cited 2020 Jun 03]. Available from: https://www.paho.org/bra/index.php ?option=com_content\&view=article\&tid $=6120$ :oms-afirma-que-covid-19-eagora-caracterizada-comopandemiact Itemid $=812$

3. World Health Organization. Genebra; 2020 [cited 2020 Jun 04]. Available from: https://covid19. who.int/

4. World Health Organization. Genebra; 2020 [cited 2020 Jun 04]. Available from:

https://covid19.who.int/region/euro/ country/pt
5. Direção-Geral da Saúde. Relatório de Situação. Lisboa; 2020 [cited 2020 Jun 04]. Available from: https: / /covid19.min-saude.pt/wpcontent/uploads/2020/06/94_DGS_bol etim_20200604.pdf

6. Wolf MS, Serper M, Opsasnick L, O'Conor RM, Curtis LM, Benavente JY, et al. Awareness, Attitudes, and Actions Related to COVID-19 Among Adults With Chronic Conditions at the Onset of the U.S. Outbreak: A Crosssectional Survey. Ann Intern Med. 2020.

7. Santos M, Fernandes MA. Internal Medicine on the Front Line: Example of a Non-Central Hospital. Rev Soc Port Med Intensiv. 2020; 20-24.

8. Almeida JA. Internal Medicine in Centro Hospitalar Universitário S. João and the COVID-19 Pandemic. Med Intensiv. 2020; 1-6.

9. Brazão ML, Nóbrega S. Internal Medicine and the COVID-19 Pandemic in Portugal. Med Intensiv. 2020; 7-9.

10. Associação Portuguesa dos Enfermeiros Gestores e Liderança (APEGEL). Editorial. Lisboa; 2020 [cited 2020 Jun 04]. Available from: http://www.apegel.org/

11. Bardin L. Análise de conteúdo. São Paulo: Edições 70; 2011. 
12. Direção-Geral da Saúde. Norma 001/2020 [Internet]. Lisboa: DireçãoGeral da Saúde; 2020. [cited 2020 Jun 05]. Available from: https://covid19.min-saude.pt/wpcontent/uploads/2020/03/i026005.p df

13. Direção-Geral da Saúde. Norma 004/2020 [Internet]. Lisboa: DireçãoGeral da Saúde; 2020 [cited 2020 Jun 06]. Available from: https://www.dgs.pt/directrizes-dadgs/normas-e-circularesnormativas/norma-n-0042020-de23032020-pdf.aspx

14. Direção-Geral da Saúde. Norma 005/2020 [Internet]. Lisboa: DireçãoGeral da Saúde; 2020 [cited 2020 Jun 04]. Available from: https://www.dgs.pt/directrizes-dadgs/normas-e-circularesnormativas/norma-n-0052020-de26032020-pdf.aspx

15. Direção-Geral da Saúde. Plano Nacional de Preparação e Resposta à Doença por novo coronavírus (COVID19) [Internet]. Lisboa: Direção-Geral da Saúde; 2020 [cited 2020 Jun 01]. Available from: https://covid19.minsaude.pt/wpcontent/uploads/2020/03/Plano-deConting\%C3\%AAncia-NovoCoronavirus_Covid-19.pdf
16. Sedes PR, Sanz MB, Saera MB, Rodríguez-Rey LC, Ortega $A C$, González MC, et al. (2020). Contingency Plan for the Intensive Care Services for the COVID-19 pandemic. Enferm Intens. 2020; 31(2):82-89.

17. Rache B, Rocha R, Nunes L, Spinola P, Malik AM, Massuda A. Necessidades de infraestrutura do SUS em preparo à COVID-19: leitos de UTI, respiradores e ocupação hospitalar [Internet]. São Paulo: Instituto de Estudos para Políticas de Saúde; 2020 [cited 2020 Jun 02]. Available from: http://www.epsjv.fiocruz.br/sites/d efault/files/files/NT3\%20vFinal.pdf

18. Direção-Geral da Saúde. Norma 007/2020 [Internet]. Lisboa: DireçãoGeral da Saúde; 2020 [cited 2020 Jun 01]. Available from: https://www.dgs.pt/directrizes-dadgs/normas-e-circularesnormativas/norma-n-0072020-de29032020-pdf.aspx

19. Ordem dos Enfermeiros. Regulamento do Perfil de Competências do Enfermeiro Gestor [Internet]. Lisboa: Ordem dos Enfermeiros; 2015 [cited 2020 May 31]. Available from: http://www.aenfermagemeasleis.pt 
/wp/wp-

content/uploads/2015/03/Regulame

nto-do-Perfil-de-

Compet\%C3\%AAncias-do-Enfermeiro-

Gestor-Ordem-dos-Enfermeiros-10-

03-2015.pdf

20. Soares SSS, Souza NVDO, Silva KG, César MP, Souto JDSS, Pereira JCRA. Covid-19 pandemic and rational use of personal protective equipment. Rev enferm UERJ. 2020; 28.

21. Direção-Geral da Saúde. Norma 012/2020 [Internet]. Lisboa: DireçãoGeral da Saúde; 2020 [cited 2020 Jun 05]. Available from: https: / /www.dgs.pt/directrizes-dadgs/normas-e-circularesnormativas/norma-n-0122020-de06052020-pdf.aspx

22. Libânio D, Bastos P, Pimentel-Nunes P. Safe and Valuable Endoscopy in the COVID Era. GE Port J Gastroenterol. 2020; 27:219-223.

23. Otero W, Gómez M, Ángel LA, Ruiz $\mathrm{O}$, Marulanda $\mathrm{H}$, Riveros $\mathrm{J}$, et al. Basic considerations regarding endoscopic procedures during the COVID-19 pandemic. Rev colomb gastroenterol. 2020; 35(1):65-75.

24. Tavares CQ. Dimensões do cuidado na perspectiva da espiritualidade durante a pandemia pelo novo coronavírus (COVID-19). J Health NPEPS. 2020; 5(1):1-4.

25. Kennedy A. Nursing the World to Health. In: International Council of Nurses. Nurses: a voice to lead nursing the world to health international nurses day 2020 resources and evidence [Internet]. Genebra: International Council of Nurses; 2020 [cited 2020 Jun 09]. p.4. Available from: https://2020.icnvoicetolead.com/wp content/uploads/2020/03/IND_Toolk it_120320.pdf

26. Viana RAPP. Enfermagem e sua atuação: a importância desta nobre profissão. J Health NPEPS. 2019; 4(2):14-15.

27. Ramos RS. Oncology Nursing in Coping with the COVID-19 Pandemic: Reflections and Recommendations for Oncology Care Practice. Rev Bras Cancerol. 2020; 66(TemaAtual):e1007.

28. Direção-Geral da Saúde. Orientação n013/2020 [Internet]. Lisboa: Direção-Geral da Saúde; 2020 [cited 2020 Jun 07]. Available from: https://www.dgs.pt/directrizes-dadgs/orientacoes-e-circularesinformativas/orientacao-n-0132020de-21032020-pdf.aspx 
29. Direção-Geral da Saúde. Orientação 018/2020 [Internet]. Lisboa: DireçãoGeral da Saúde; 2020 [cited 2020 Jun 10]. Available from: https://covid19.min-saude.pt/wpcontent/uploads/2020/06/i026356.p $\mathrm{df}$

30. Rondelli G, Jardim D, Hamad G, Luna E, Marinho W, Mendes L, et al. Assistência às gestantes e recémnascidos no contexto da infecção COVID-19: uma revisão sistemática. Rev Desafios. 2020; 7(Especial-3):4874.

31. Direção-Geral da Saúde. Norma 008/2020 [Internet]. Lisboa: DireçãoGeral da Saúde; 2020 [cited 2020 Jun 05]. Available from: https://www.dgs.pt/directrizes-dadgs/normas-e-circularesnormativas/norma-n-0082020-de280320201.aspx

32. Domingos A, Guedes AM, Neves PL. Peritoneal Dialysis in the Current Pandemic Crisis: Na Opportunity for Reflection. Acta Med Port. 2020; 33(5):357-358.

33. Direção-Geral da Saúde. Norma 009/2020 [Internet]. Lisboa: DireçãoGeral da Saúde; 2020 [cited 2020 Jun
09]. Available from: https://www.dgs.pt/directrizes-dadgs/normas-e-circularesnormativas/norma-n-0092020-de02042020-pdf.aspx

34. Direção-Geral da Saúde. COVID-19: Orientações para os hospitais. Lisboa; 2020 [cited 2020 Jun 13]. Available from: https://covid19.minsaude.pt/covid-19-orientacoes-paraos-hospitais/

35. Dantas TP, Aguiar CAS, Rodrigues VRT, Silva RRG, Silva MIC, Sampaio LRL, et al. Diagnósticos de enfermagem para pacientes com COVID-19. J Health NPEPS. 2020; $5(1): 396-416$.

36. Mendonça FD, Rocha SS, Pinheiro DLP, Oliveira SV. Região Norte do Brasil e a pandemia de COVID-19: análise socioeconômica e epidemiológica. J Health NPEPS. 2020; 5(1):20-37.

Conflito de interesses: Os autores declaram não haver conflito de interesses. 
Participação dos autores:

- Concepção: Ventura-Silva JMA, Ribeiro OMPL, Santos MR, Faria ACA, Monteiro MAJ, Vandresen L.

- Desenvolvimento: Ventura-Silva JMA, Ribeiro OMPL, Santos MR.

- Redação e revisão: Ventura-Silva JMA, Ribeiro OMPL, Santos MR, Faria ACA, Monteiro MAJ, Vandresen L.

Como citar este artigo: Ventura-Silva JMA, Ribeiro OMPL, Santos MR, Faria ACA, Monteiro MAJ, Vandresen L. Planejamento organizacional no contexto de pandemia por COVID-19: implicações para a gestão em enfermagem. J Health NPEPS. 2020; 5(1):e4626.

Submissão (Fast Track COVID-19): 18/06/2020

Aceito (Fast Track COVID-19): 29/06/2020

Publicado (Fast Track COVID-19): 29/06/2020 